Theophilus Lazarus

Emory University,

Atlanta, GA, United States;

Prof T Lazarus Inc., Neuropsychology Practice,

Durban, South Africa

\title{
Using Luria's neuropsychological approach to functional brain organization to understanding epilepsy
}

Abstract: Seizures and epilepsy comprise disorders of the brain in which there are abnormal discharges of the brain cells (neurons) resulting in various observable behavioural disorders. Whilst the basic underlying neuropathology of these disorders is the same in all individuals, the manifestations in cognition, intellect, emotion, socialization and behaviour have variations across individuals.

Keywords: Epilepsy; Luria; Seizures; Neuron; Abnormal discharge; Behavioural, Cognitive and Emotional Disorders

Introduction. Seizures and epilepsies result from abnormal discharges of the brain. The abnormal neuronal discharge may be localized to one area of the brain or may generalize to the entire brain within and across hemispheres, provoking a varied pattern of cognitive and behavioural disturbances. Seizure classification systems were developed by the International League Against Epilepsy [1] and are generally distinguished as focal or generalized in nature. Behavioral symptoms may be localized to a specific area and generally fit into a partial or generalized category involving various patterns of behavioural, cognitive and motor symptoms. The behavioural manifestations which are usually observed, first by family or other people close to the patient, form a critical part or the diagnostic process in arriving at a specific type of epilepsy type. The advancement in neuroimaging, genetics and molecular biology is incorporated into identifying the seizure and epilepsy types and syndromes. Social and cultural contributions to describing and understanding of seizure phenomena play an important role in managing these problems. Therefore, the approach to neuropsychology of epilepsy proposed by Luria [2] in his 
Syndrome Analysis, as articulated by Glozman [3] and Zinchenko \& Pervichko [4], focuses on observing and analyzing the structure of each administered psychological task, the types of errors that are produced, and the anticipation of conditions that minimize or overcome the identified deficits. Such approach affords a useful way of discussing and managing individual cases of epilepsy.

Materials and Methods. The patient of interest is a young woman, a nursing sister 28 years of age, initially diagnosed with pulmonary sarcoma that later metastasized to the right cerebral hemisphere. Sarcoidosis is a chronic inflammatory disorder. A Magnetic Resonance Imaging investigation revealed evidence of generalized cerebral atrophy that was especially pronounced in relation to temporal lobes with bilateral multiple white matter hyper-intensities noted bilaterally and prominence of $4^{\text {th }}$ and supratentorial ventricular system that was consistent with cerebral atrophy. In addition, a degree of cerebellar atrophy was found. Apart from complaints of muscle weakness and headaches, as well optic disturbances, the patient was diagnosed with epileptic seizures that required management with Levetiracetam (trade name Keppra, 500mg taken twice daily). The patient reported cognitive problems that affected her work and daily living. Since the cognitive effects of neurosarcoidosis are poorly understood with limited research reported [5], the patient underwent comprehensive neuropsychological assessment that included the Luria Neuropsychological Assessment and Syndrome Analysis.

Results. Neuropsychological testing revealed overall low intellectual function, a probably decline from the estimated premorbid average range of intellectual functioning that was obtained from previous educational records. Significant syndrome inefficiencies were found in the domains of information processing speed, executive function (forming concepts, tracking and in verbal and visual abstraction abilities), auditory and visual memory (both words and narrative information), fluent language and in visuospatial organization of information. These findings are concordant with those reported by Pruter, Kunert, \& Hoff [5]. However, the findings suggesting emotional disinhibition with irritability and anger may form another Luria Syndrome relating to Frontal Disinhibition [2]. Most important were the breakthrough seizures that the patient's spouse observed nocturnally with teeth biting, mild head jerking, mild 
groaning sounds and amnesia for the events when she was questioned the next morning.

Conclusions. The findings of this case study, using Luria's Syndrome Analysis, highlight the usefulness of this assessment approach when investigating seizure and epilepsy patients with a positive medical history for neuropathology when making decisions for medical control and for recommendations in regard to self-care and work activities. Luria's Syndrome Analysis makes possible an analysis of underlying cognitive and emotional-behavioural problems that are unique to the patient's sociocultural history and therefore provides a basis for sound recommendations in the management of seizures and epilepsy and for daily activities.

1. Scheffer IE, Berkovic S, Capovilla G, et al. ILAE classification of the epilepsies: Position paper of the ILAE Commission for Classification and Terminology // Epilepsia. 2017. 58(4):512-521.

2. Luria A. Higher cortical functions in man. New York: Basic Books, 1966.

3. Glozman J.M. Quantitative and Qualitative Integration of Lurian Procedures // Neuropsychological Review. 1999. № 9. P. 23-32.

4. Zinchenko Y.P., Pervichko E. I. The Methodology of Syndrome Analysis within the Paradigm of "Qualitative Research" in Clinical Psychology // Psychology in Russia: State of the Art. 2012. № 5(1). P. 157-184.

5. Pruter C., Kunert H. J., Hoff P. ICD-10 mild cognitive disorder following meningitis due to neurosarcoidosis // Psychopathology. 2001. № 34. P. 326-327. 